\title{
Intrinsic transverse momentum and transverse spin asymmetries
}

\author{
Daniël Boer \\ RIKEN-BNL Research Center \\ Brookhaven National Laboratory, Upton, NY 11973, U.S.A.
}

\begin{abstract}
We investigate leading twist transverse momentum dependent origins of transverse spin asymmetries in hadron-hadron collisions. The chiral-odd T-odd distribution function with intrinsic transverse momentum dependence, which would signal an intrinsic handedness of quarks inside a hadron, could account for single spin asymmetries and at the same time for the large $\cos 2 \phi$ asymmetry in the unpolarized Drell-Yan cross section, which still lacks understanding. We show explicitly how it would relate unpolarized and polarized observables measurable with proton-proton collisions at RHIC. It would offer a new possibility to access the transversity distribution function.
\end{abstract}

Talk given at the 7th International Workshop on

"Deep Inelastic Scattering and QCD" (DIS99)

DESY-Zeuthen, April 19 - 23, 1999 


\title{
Intrinsic transverse momentum and transverse spin asymmetries
}

\author{
Daniël Boer ${ }^{\mathrm{a}}$ \\ ${ }^{a}$ RIKEN-BNL Research Center, Brookhaven National Laboratory, Upton, NY 11973, U.S.A.
}

\begin{abstract}
We investigate leading twist transverse momentum dependent origins of transverse spin asymmetries in hadronhadron collisions. The chiral-odd T-odd distribution function with intrinsic transverse momentum dependence, which would signal an intrinsic handedness of quarks inside a hadron, could account for single spin asymmetries and at the same time for the large $\cos 2 \phi$ asymmetry in the unpolarized Drell-Yan cross section, which still lacks understanding. We show explicitly how it would relate unpolarized and polarized observables measurable with proton-proton collisions at RHIC. It would offer a new possibility to access the transversity distribution function.
\end{abstract}

\section{Introduction}

Large single transverse spin asymmetries have been observed in the process $p p^{\uparrow} \rightarrow \pi X[$ [ ]. Of course, one experiment only cannot reveal the origin(s) of such asymmetries conclusively and one needs comparison to other experiments, for instance the planned RHIC spin physics experiments.

The transverse momentum dependence of transverse spin asymmetries should be related to the transverse momentum of quarks inside a hadron. Our goal is to investigate the relation between the transverse spin and transverse momentum of quarks.

In Ref. [ 2] we have argued that conventional perturbative QCD and higher twist effects do not produce large -if any- single transverse spin asymmetries. Less conventional higher twist mechanisms, such as soft gluon poles in twist-3 matrix elements [3] or the effectively equivalent twist-3 T-odd distribution functions [ [4], could produce a single spin asymmetry. For the DrellYan (DY) process it is expected to be similar in size to the double spin asymmetry $A_{L T}$ [ [5], which is estimated [6] to be much smaller than the double transverse spin asymmetry $A_{T T}$, that is estimated to be on the order of percents for RHIC energies [ 7]. Therefore, we proposed an alternative explanation of such a single spin asymmetry, involving a particular leading twist, intrinsic transverse momentum dependent, chiral-odd, T-odd distribution function [ 8], called $h_{1}^{\perp}$ (cf.
Fig. 1: depicted are probabilities of specific quark states (black dot) inside a hadron). This can not only offer an explanation for single spin asymmetries in $p p^{\uparrow} \rightarrow \pi X$ or the DY process, but also for the large azimuthal $\cos 2 \phi$ dependence of the unpolarized DY cross section [9, 10], which still lacks understanding.

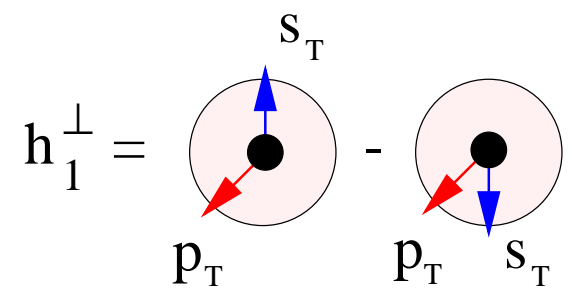

Figure 1. The chiral-odd T-odd distribution function $h_{1}^{\perp}\left(s_{T}\right.$ and $p_{T}$ are the quark's transverse spin and momentum).

Unlike its chiral-even counterpart $f_{1 T}^{\perp}$ (investigated in [11, 12]), which depends on the polarization of the parent hadron (cf. Fig. 2), the function $h_{1}^{\perp}$ signals an intrinsic handedness inside an unpolarized hadron. It would mean an orientation dependent correlation between the transverse spin and the transverse momentum of quarks inside an unpolarized hadron [. One can use the

${ }^{1}$ In Ref. [2] we have discussed the theoretical difficulties associated with T-odd distribution functions. 


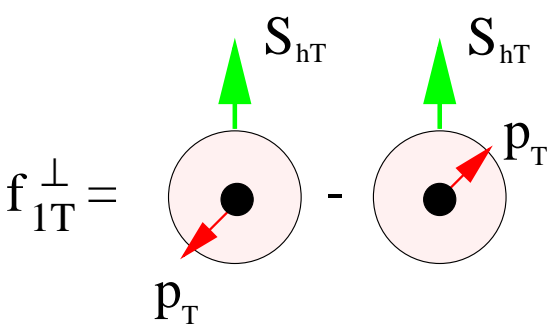

Figure 2. The chiral-even T-odd distribution function $f_{1 T}^{\perp}\left(S_{h T}\right.$ is the hadron transverse spin).

polarization of another hadron to become sensitive to the polarization of quarks inside an unpolarized hadron. In this way it could provide a new way of measuring the transversity distribution function $h_{1}$. For this purpose we propose two measurements that could be done at RHIC using polarized proton-proton collisions.

\section{An unpolarized asymmetry}

A large $\cos 2 \phi$ angular dependence in the unpolarized DY process $\pi^{-} N \rightarrow \mu^{+} \mu^{-} X$, where $N$ is either deuterium or tungsten and for instance using a $\pi^{-}$beam of $194 \mathrm{GeV}$, was found by the NA10 Collaboration [9]. The perturbative QCD prediction (NLO) for the cross section written as

$\frac{d \sigma}{d \Omega} \propto 1+\cos ^{2} \theta+\sin ^{2} \theta\left[\mu \cos \phi+\frac{\nu}{2} \cos 2 \phi\right]$,

is $\mu \approx 0, \nu \approx 0$. However, $\nu$ acquires values of more than 0.3 depending on the transverse momentum $Q_{T}$ of the muon pair (its invariant mass is between 4 and $8 \mathrm{GeV} / c^{2}$ ), cf. Fig. 3. Even though the cross section itself is dependent on the nuclear target, since $\sigma_{W}\left(Q_{T}\right) / \sigma_{D}\left(Q_{T}\right)$ is an increasing function of $Q_{T}$, the analyzing power $\nu\left(Q_{T}\right)$ shows no apparent nuclear dependence, indicating that the asymmetry arises at the quark-hadron level.

In Ref. [2] we have observed that within the framework of transverse momentum dependent distribution functions [13, 8], this asymmetry can only be accounted for by the function $h_{1}^{\perp}$, unless $1 / Q^{2}$ suppressed [ 14. Moreover, higher twist effects are expected to produce $\mu>\nu$, which is not the case. We found $\nu \propto h_{1}^{\perp} \pi h_{1}^{\perp}$ and used this observation to fit the function $h_{1}^{\perp}$, assuming some simplifications, like independence of the type of parent hadron, cf. Fig. 3. In a sim-

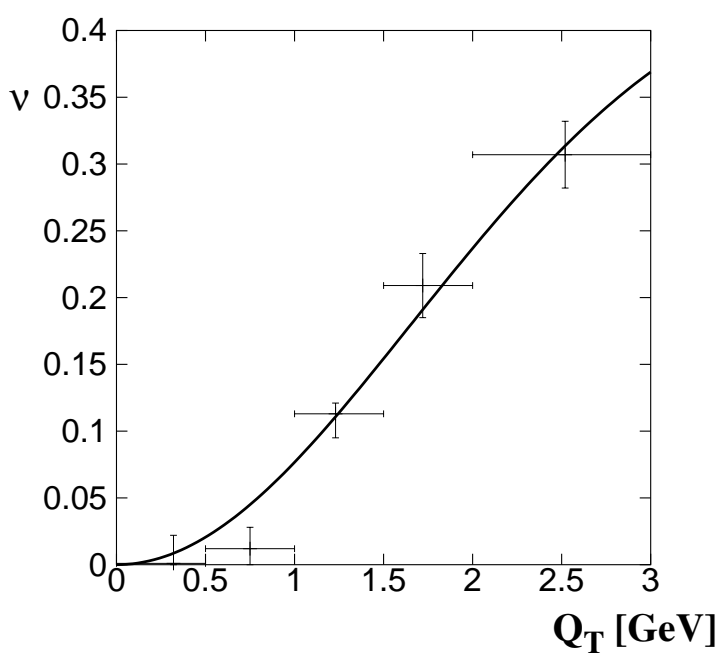

Figure 3. Data from [ 9] at $194 \mathrm{GeV}$ and fit to $\nu$ as a function of the transverse momentum $Q_{T}$ of the lepton pair.

ilar way one can try to measure $\langle\cos 2 \phi\rangle$ in unpolarized $p p \rightarrow \mu^{+} \mu^{-} X$ at RHIC and obtain a parametrization of $h_{1}^{\perp p}$.

\section{Single spin asymmetries}

At RHIC they will also be able to measure $p p^{\uparrow} \rightarrow \mu^{+} \mu^{-} X$. With one transversely polarized hadron the DY cross section will have more complicated azimuthal dependences. For instance (displaying only two terms):

$\frac{d \sigma}{d \Omega d \phi_{S_{T}}} \propto \sin ^{2} \theta\left[\frac{\nu}{2} \cos 2 \phi-\rho \sin \left(\phi+\phi_{S_{T}}\right)\right]$.

The analyzing power $\rho$ is proportional to the product $h_{1}^{\perp} h_{1}$ [2]. Hence, the measurement of $\langle\cos 2 \phi\rangle$ combined with a measurement of the single spin azimuthal asymmetry $\left\langle\sin \left(\phi+\phi_{S_{T}}\right)\right\rangle$ could provide information on $h_{1}$. In other words, a nonzero function $h_{1}^{\perp}$ will imply a relation be- 
tween $\nu$ and $\rho$, which in case of one flavor is $(\nu \max$ is the maximum value attained by $\nu\left(Q_{T}\right)$ )

$\rho=\frac{1}{2}\left|\boldsymbol{S}_{T}\right| \sqrt{\frac{\nu}{\nu_{\max }}} \frac{h_{1}}{f_{1}}$.

This depends on the magnitude of $h_{1}$ compared to $f_{1}$ and in Fig. A 1 we display three options for $\rho$, using the fitted function $\nu$ which we view as an optimistic upper bound.

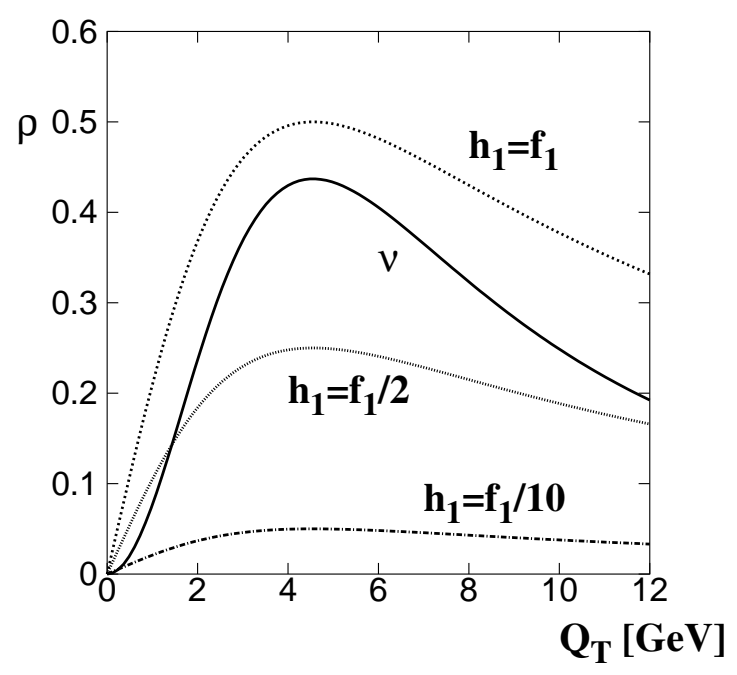

Figure 4. Upper bound predictions for $\rho$ for $h_{1} / f_{1}=\{1,1 / 2,1 / 10\}$, using the fit for $\nu$.

We note that the function $f_{1 T}^{\perp}$ generates a totally different angular single spin asymmetry, namely $\left(1+\cos ^{2} \theta\right)\left|\boldsymbol{S}_{T}\right| \sin \left(\phi-\phi_{S_{T}}\right) f_{1 T}^{\perp} f_{1}$.

The large single spin asymmetries found in $p p^{\uparrow} \rightarrow \pi X$ can also arise from leading twist $\mathrm{T}$ odd functions with transverse momentum dependence. There are three options:

$$
\begin{aligned}
& h_{1}^{\perp}\left(x_{1}, \boldsymbol{p}_{T}\right) \otimes h_{1}\left(x_{2}\right) \otimes D_{1}(z), \\
& f_{1 T}^{\perp}\left(x_{1}, \boldsymbol{p}_{T}\right) \otimes f_{1}\left(x_{2}\right) \otimes D_{1}(z), \\
& h_{1}\left(x_{1}\right) \otimes f_{1}\left(x_{2}\right) \otimes H_{1}^{\perp}\left(z, \boldsymbol{k}_{T}\right) .
\end{aligned}
$$

The first two options are similar to the ones described above, accompanied by the unpolarized fragmentation function $D_{1}$. The third option contains the Collins effect function $H_{1}^{\perp}$ [15], which is formally the fragmentation function analogue of $h_{1}^{\perp}$, but in principle unrelated in magnitude. The last two options were investigated in [12].

\section{Conclusion}

The chiral-odd T-odd distribution function $h_{1}^{\perp}$ can not only offer an explanation for single transverse spin asymmetries in hadron-hadron collisions, but also for the unpolarized $\cos 2 \phi$ asymmetry in the $\pi^{-} N \rightarrow \mu^{+} \mu^{-} X$ data (unlike any other function in this approach, unless $1 / Q^{2}$ suppressed). It would relate unpolarized and polarized observables and thus would offer a new possibility to access $h_{1}$ in $p p \rightarrow \mu^{+} \mu^{-} X$.

\section{REFERENCES}

1. D.L. Adams et al., Phys. Lett. B 264 (1991) 462; A. Bravar et al., Phys. Rev. Lett. 77 (1996) 2626.

2. D. Boer, hep-ph/9902255.

3. J. Qiu, G. Sterman, Phys. Rev. Lett. 67 (1991) 2264; Phys. Rev. D 59 (1999) 014004; N. Hammon, O. Teryaev, A. Schäfer, Phys. Lett. B 390 (1997) 409.

4. D. Boer, P.J. Mulders, O.V. Teryaev, Phys. Rev. D 57 (1998) 3057.

5. R.L. Jaffe, X. Ji, Nucl. Phys. B 375 (1992) 527.

6. Y. Kanazawa, Y. Koike, N. Nishiyama, Phys. Lett. B 430 (1998) 195.

7. O. Martin et al., hep-ph/9902250.

8. D. Boer, P.J. Mulders, Phys. Rev. D 57 (1998) 5780 .

9. S. Falciano et al., Z. Phys. C 31 (1986) 513; M. Guanziroli et al., Z. Phys. C 37 (1988) 545.

10. J.S. Conway et al., Phys. Rev. D 39 (1989) 92.

11. D. Sivers, Phys. Rev. D 41 (1990) 83.

12. M. Anselmino, M. Boglione, F. Murgia, Phys. Lett. B 362 (1995) 164; hepph/9901442.

13. R.D. Tangerman, P.J. Mulders, Phys. Rev. D 51 (1995) 3357.

14. E.L. Berger, Z. Phys. C 4 (1980) 289.

15. J.C. Collins, Nucl. Phys. B 396 (1993) 161. 